\title{
Territorialidad y reforma al sector seguridad: reflexiones conceptuales y experiencias desde la Policía Nacional en Colombia ${ }^{1}$
}

https://doi.org/10.21830/9789585350601.04

\author{
Mateo Morales Callejas ${ }^{2}$ \\ Universidad Militar Nueva Granada
}

\section{Resumen}

El capítulo tiene el propósito de generar una reflexión sobre la reforma al sector seguridad en clave territorial. A partir de una reflexión conceptual en torno al enfoque de paz territorial y a la manera como dicho enfoque es clave de cara a la gestión de la seguridad y la convivencia en el posacuerdo, se desarrollan las experiencias de un proyecto de la Policía Nacional circunscrito al Modelo de Construcción de Paz de la institución. El capítulo pone en evidencia los principales retos en materia de territorializar los temas de seguridad y convivencia ciudadana, y sugiere prestar atención a los avances llevados a cabo por la Policía, de modo tal que puedan ser replicados en procesos más amplios de reforma del sector seguridad y defensa en Colombia.

Palabras clave: Colombia; convivencia; paz territorial; Policía Nacional; posacuerdo; seguridad.

\section{Introducción}

Los acuerdos de paz negociados en La Habana entre el gobierno de Juan Manuel Santos (2010-2018) y las Fuerzas Armadas Revolucionarias de Colombia (FARC), firmados en el Teatro Colón en 2016, y el subsecuente Acto

1 Este capítulo es producto de investigación del proyecto INV-EES 2975 de 2019 "El panorama del post conflicto en Colombia: un acercamiento desde la gobernanza y la reforma del sector seguridad", financiado por la Universidad Militar Nueva Granada. Se reconoce la participación de la Universidad del Rosario en el marco del Convenio Especial de Cooperación con la Universidad Militar Nueva Granada.

2 Politólogo y magíster en Ciencia Política de la Universidad de los Andes. Orcid: https://orcid. org/0000-0001-5272-1959. 
Legislativo 01 de 2017, por el cual "se crea un título de disposiciones transitorias de la Constitución para la terminación del conflicto armado y la construcción de una paz estable y duradera”, tienen como asunto esencial el territorio. Esto se visibiliza de varias formas, pero especialmente en el Sistema Integral de Verdad, Justicia, Reparación y No Repetición. Este sistema tiene un "enfoque territorial, diferencial y de género" que responde a las características específicas de la victimización de cada territorio y cada población, al tiempo que presta atención particular a la "protección y atención prioritaria de las mujeres y de los niños y nińas víctimas del conflicto armado”, con lo cual da respuesta al enfoque de paz territorial que se tuvo en las negociaciones y que existe en la implementación de los acuerdos.

Si bien el acuerdo de paz no generó unas revisiones sustanciales al ordenamiento político y económico del país, su enfoque de paz territorial sí marca un punto de quiebre de relevancia para abordar el conflicto armado, teniendo en cuenta las enormes desigualdades que se presentan a lo largo y ancho de la geografía nacional, y que este enfoque pretende abordar por medio de los diferentes programas destinados para ello, por ejemplo los Programas de Desarrollo con Enfoque Territorial (РDET).

La desmovilización de la guerrilla más fuerte del país sin duda ofreció la oportunidad de copar los territorios anteriormente dominados por este actor y comenzar a impulsar una agenda de desarrollo territorial, con lo cual se comienza a saldar la deuda histórica que el Estado colombiano ha tenido frente a lo que se conoce como la Colombia profunda. Sin embargo, este no fue el caso, ya que en gran medida los territorios controlados por las FARC comenzaron a ser disputados y gobernados por otros actores armados ilegales que les sucedieron, en función de controlar las rentas ilegales que la minería ilegal y el narcotráfico producen en estos territorios. Sin embargo, más allá de los beneficios económicos, estos grupos han ganado su institucionalidad y legitimidad gracias a la resolución de disputas locales y la defensa de los medios de vida ilícitos frente a las fuerzas de seguridad del Estado, con lo cual han establecido una forma de liderazgo político local basado en la coerción (International Crisis Group, 2017).

En ese sentido, lo que se aprecia actualmente es que se ha desaprovechado una oportunidad única para poder llegar a los territorios y comenzar a generar 
procesos de desarrollo e inclusión, además de abordar de manera diferenciada las necesidades locales que sobrepasan por mucho la presencia militar y policial del Estado. En sus relaciones con la sociedad civil en materia de garantías de seguridad y convivencia ciudadana se relegan a un segundo plano, lo que da paso a la legitimación de la acción de los grupos al margen de la ley que dan garantía de estas demandas. Del mismo modo, el frecuente enfrentamiento entre grupos alzados en armas y la Fuerza Pública ha generado tensiones entre las comunidades, de modo que esta última ha llegado a considerar la mayor presencia del Estado como una amenaza a su seguridad (Llorente et al., 2016).

Lo anterior constituye un gran pendiente en materia de seguridad y convivencia ciudadana por parte de la Fuerza Pública y del Estado, de tal manera que se requiere, según los procesos territoriales existentes, un abordaje que sobrepase el uso del aparato coercitivo para el control territorial. Asimismo, es necesario desarrollar un enfoque participativo que involucre liderazgos territoriales que permitan articular de manera efectiva las demandas sociales en materia de seguridad y convivencia (Llorente et al., 2016), con lo cual se da paso a un nuevo proceso de organización, intervención y acompañamiento mutuo en el territorio que da lugar al apoyo y la legitimidad de la acción estatal por parte de las comunidades.

Con el fin de hacer frente a estas problemáticas, se han generado una serie de reformas en las Fuerzas Militares y de Policía (incluso antes de la firma del acuerdo) para hacer frente a las dinámicas territoriales (ya existentes y nuevas), las cuales tienen como fundamento la experiencia adquirida en los más de cincuenta años de conflicto. Entre estas reformas se destacan la Doctrina Damasco en el Ejército Nacional (Rojas, 2017) y el Modelo de Construcción de Paz de la Policía Nacional (Unidad Policial para la Edificación de la Paz [Unipep], 2017). Pese a la existencia de este tipo de innovaciones institucionales, se aprecia que estas no se encuentran empaquetadas en una clara política nacional frente a los temas de seguridad y defensa, sino que, por el contrario, han surgido del actuar autónomo de las mismas instituciones.

Un claro ejemplo de lo anterior son los proyectos que han surgido en el seno del Modelo de Construcción de Paz de la Policía, los cuales se han materializado con la ayuda de la cooperación internacional. En términos de 
visibilizar la importancia de lo territorial, la UNIPEP desarrolló una experiencia que tuvo como propósito comenzar a visibilizar esas problemáticas que van más allá de la conflictividad armada, se asientan a nivel comunitario y cuyo abordaje es determinante para prevenir escalamientos de los conflictos. En términos generales, este proyecto, bautizado con el nombre de "Suma: voces y acciones para la convivencia", se ha constituido como un referente institucional, dado que no solo se puede leer como una manifestación del concepto de paz territorial, sino que además involucra toda una serie de principios y enfoques que hasta hace muy poco eran impensables para una institución como la Policía, volcada casi exclusivamente hacia los asuntos de seguridad pública (Policía Nacional, 2019).

En ese orden de ideas, el presente capítulo tiene el objetivo de desarrollar una reflexión en torno a la relevancia del territorio como parte integral de cualquier proceso de construcción de paz y sobre cómo esta variable territorial está comenzando a ser integrada por instituciones como la Policía dentro de su propio proceso de reforma de cara a un posconflicto. Para ello, el capítulo comienza profundizando sobre el concepto de paz territorial, para, seguidamente, mostrar de qué manera los espacios generados por la firma del acuerdo de paz fueron subutilizados y dieron cabida al ingreso de nuevos actores armados que han impedido la consolidación integral del enfoque. Posteriormente, se describen los procesos de reforma de las instituciones de seguridad para poner en evidencia su relevancia y dar luces sobre algunas problemáticas que se presentan de cara a generar una reforma real del sector frente a los territorios. Finalmente, se analiza la experiencia policial para evidenciar cómo este tipo de iniciativas materializan el enfoque de paz territorial y fomentan la construcción a nivel micro de geografías de paz.

El énfasis que se hace en la institución policial, tanto en la reflexión teórico-conceptual como en la experiencia como tal, se debe a dos razones que vale la pena resaltar. Por una parte, a diferencia de las Fuerzas Militares, la Policía Nacional ha desarrollado y gestionado un programa de construcción de paz mediante el cual se están materializando las reformas institucionales, mientras que, a la fecha, no es de público conocimiento un modelo similar del lado militar. Por otra parte, la información disponible sobre las experiencias de 
la Policía es mucho mayor, tanto documental como de primera mano, teniendo en cuenta que gran parte de los proyectos que se realizan desde el Modelo de Construcción de Paz de la Policía se ejecutan a través de la Organización Internacional para las Migraciones, lo cual permite el acceso a documentación de proyecto, informes y documentos de sistematización.

\section{Paz territorial y la importancia de lo local}

La perspectiva de paz territorial responde principalmente a un enfoque territorial de las políticas públicas necesarias para implementar los acuerdos. El enfoque territorial está directamente relacionado con el desarrollo, que se puede lograr por medio de la articulación estratégica de acciones e instituciones para producir cambios a las estructuras socioeconómicas y ambientales, así como para reconfigurar las políticas públicas.

Este enfoque posee las siguientes características: (1) la multidimensionalidad, vista como el entendimiento de la interacción territorial entre lo político-institucional, lo ambiental y lo social; (2) la multisectorialidad, la cual se refiere a que en las zonas rurales o en el conjunto de regiones se desarrollan diversas actividades económicas que pueden abarcar varios sectores, como la agricultura, las artesanías, las industrias pequeñas y medianas, el comercio, los servicios, la ganadería, el turismo, la extracción de recursos naturales, etc.; (3) la capitalización humana, social y natural, que consiste en el potencial para influir en la configuración del territorio que tienen los capitales humanos (la capacidad de las personas), sociales (las relaciones y redes que facilitan la gobernabilidad) y de los recursos naturales; (4) la articulación urbano-rural, que no solo examina la necesidad de coordinar las dinámicas urbanas y rurales, sino que además destaca la importancia de las políticas de ordenamiento territorial, autonómica y de autogestión como complementos a las políticas de descentralización y participación ciudadana; (5) el valor agregado territorial, el cual reconoce la importancia de los encadenamientos productivos que generan valor agregado, existentes o potenciales, entre los diferentes territorios y sectores económicos; (6) la diferenciación territorial, que busca la promoción y creación de esquemas de cooperación que se adapten a las variadas demandas 
de las poblaciones presentes en los territorios, teniendo como base la heterogeneidad económica, política y cultural de las dinámicas territoriales que demandan estrategias diferenciadas; (7) la articulación territorial, que observa a los territorios como unidades interrelacionadas entre sí en un proceso social y cultural más amplio, el cual se asienta sobre bases estructurales de extracción, producción, comercialización y consumo que son armonizadas por instituciones legales o ilegales que pueden ser de orden supranacional, nacional o local (Calvo, 2005, p. 3; Guarín, 2016).

El enfoque territorial contribuye a la percepción holística del territorio, ayudando a establecer una visión estratégica y sistemática de acción para un corto, mediano y largo plazo en la intervención territorial. En este sentido, la planificación tiene un papel fundamental para solventar los problemas a nivel local y aprovechar de manera adecuada las potencialidades de los procesos económicos, políticos, sociales, ambientales y culturales presentes en el territorio (Carvajal, 2017). En este sentido, es imprescindible dejar a un lado la acepción histórica corriente en la formulación de políticas o programas según la cual se busca el desarrollo únicamente mediante procedimientos que promuevan el crecimiento del sector primario-, en beneficio de acciones que contribuyan a la evolución integral de los diferentes procesos (Presidencia de la República et al., 2019).

Asimismo, respecto a la presencia del Estado en el territorio colombiano es importante resaltar que históricamente ha sido desigual, pues ha estado ausente en muchas zonas de carácter rural con mayor grado de afectación del conflicto. Esta situación ha derivado en la incapacidad institucional estatal de hacer cumplir la ley, tramitar conflictos de la ciudadanía y promover un orden social que garantice los derechos y las pautas de convivencia. Como consecuencia, se han otorgado espacios para que existan múltiples órdenes que compiten o sustituyen, en ocasiones, el control del Estado en el territorio (Llorente et al., 2016), los cuales cuentan con legitimidad por parte de las poblaciones locales. Se trata de una situación trascendental en el posconflicto, puesto que, como señalan García y Espinosa (2013, p. 14), “estas zonas de apartheid institucional ocupan más del $60 \%$ del territorio nacional y en ellas viven más de seis millones de personas". 
En este sentido, cuando se habla de la paz territorial, no solo se debe hacer referencia a la interconexión territorial que debe existir y a la necesidad de priorizar el desarrollo local para integrar a estos territorios que tradicionalmente han sido olvidados de las dinámicas nacionales de crecimiento, sino también hacer un abordaje desde la óptica de la seguridad y de los conflictos. $\mathrm{Si}$ estas condiciones de base son débiles, el desarrollo económico será igualmente inestable y potencialmente susceptible de ser cooptado o desestabilizado por las agendas de los actores ilegales que pueden estar presentes en los territorios. En gran medida, la existencia de territorios seguros y donde haya convivencia pacífica entre sus comunidades es una precondición para que se pueda dar de manera integral una paz territorial, según como quedó estipulada con la firma del acuerdo.

Para el éxito de la paz en el país es indispensable hacer gobernable el posacuerdo en el territorio, de manera que se requiere fortalecer las instituciones del Estado y asegurar mecanismos de gobernabilidad local, con una estructura nacional coherente y sobre la base de erradicar la desconfianza mutua entre el Estado y la comunidad. En algunas localidades, la comunidad no se acerca a las instituciones estatales por medio de los mecanismos de tramitación de conflictos establecidos porque considera que el Estado ve a las poblaciones como un apéndice de la insurgencia o como una amenaza.

Lo anterior implica, por lo tanto, que la consolidación de una paz territorial en su componente socioeconómico, pero también de seguridad, debe ser sensible a las realidades locales en muy variados aspectos, por ejemplo en la heterogeneidad territorial y temporal del conflicto armado; los modos de inserción y de relacionamiento de los actores armados con las comunidades, la presencia diferenciada del Estado y la existencia o ausencia de capacidades locales endógenas. Este último aspecto es de gran relevancia y ha tendido a ser descuidado por los diferentes análisis que se hacen respecto de lo local. El conflicto no solo creó anomia o destrucción del tejido social, sino que también creó capacidades endógenas a las mismas comunidades, que les han permitido tener capacidad de adaptación frente a los actores armados. Estas capacidades endógenas no pueden o no deben ser descuidadas a la hora de planificar la implementación de una paz en el territorio, pues en gran medida son las que 
determinarán si lo que se está haciendo es, en efecto, sensible a las necesidades de las poblaciones. Esto será retomado con más detalle cuando se aborde el caso de la Policía.

\section{Conflicto armado y crimen organizado que persiste}

El final de la Guerra Fría trajo consigo un aumento en los conflictos intra-estatales o, mejor, una mayor visibilización de estos fenómenos. Hasta el momento el grueso de las explicaciones y de la atención académica había estado puesta en comprender y explicar el conflicto bipolar entre las superpotencias, tratando de predecir su desenlace. Sin embargo, una vez se disolvió la tensión entre Estados Unidos y la Unión Soviética, ocurrido por lo demás de forma pacífica, se evidencia que hubo un cambio en la atención de los académicos, quienes se enfocaron en estudiar los conflictos internos, sus causas, consecuencias y lógicas.

Autores como Ann Hironaka (2005) han mostrado por medio del estudio estadístico de múltiples casos que no solo los Estados débiles tienden a ser mayores generadores de conflictos internos, sino que estos conflictos tienden a ser más duraderos por el hecho de que las unidades políticas carecen de recursos y capacidades organizacionales para ponerle fin a los enfrentamientos. De manera específica, argumenta que existen factores que impiden llegar a una terminación pronta, tales como la influencia de ciertos grupos políticos de la sociedad que impiden las negociaciones con los grupos armados; la falta de capacidades militares por parte del Estado para derrotar a sus oponentes; la falta de control del Estado de sus fronteras y su territorio, y la existencia de sociedades divididas, lo cual a su vez engendra múltiples grupos rebeldes frente a los cuales es imposible dirigir acciones militares coordinadas o establecer negociaciones vinculantes (Hironaka, 2005, pp. 69-70).

$\mathrm{Al}$ analizar el estado actual del orden público a nivel nacional, lo que se aprecia es precisamente la incapacidad del Estado de copar los territorios dejados por las FARC, así como la proliferación de otros actores que de manera eficaz han sido capaces de controlar estos territorios, sus poblaciones y las economías ilegales que persisten. Esto ha desencadenado nuevas oleadas de 
violencia, sobre todo en contra de líderes sociales y defensores de Derechos Humanos, quienes, con el entendido de que el conflicto con las Farc había desaparecido, comenzaron a abogar por la defensa y garantía de los derechos que por décadas habían sido negados a raíz del conflicto armado. Sin embargo, dada la ausencia de una efectiva institucionalidad, estos líderes se convirtieron en blancos fáciles dada la ausencia de garantías de seguridad por parte del Estado.

El reagrupamiento y tránsito a la legalidad de las FARC derivó en la generación de espacios vacíos de poder local, donde las FARC tenían el control territorial y legitimidad en su acción, y donde existe una histórica ausencia estatal (Lemaitre \& Restrepo, 2019). Como resultado de estos vacíos de poder en los 242 municipios donde tenían presencia y control las FARC, se produjo una ventana de oportunidad tanto para el Estado como para los demás grupos criminales, razón por la cual se presentó alguna de las siguientes situaciones: (1) muchas zonas quedaron en el limbo e inclusive se detuvo la compra de pasta base de coca a los campesinos, debido a que no se contaba con la seguridad garantizada por las FARC. Allí se esperaba la presencia estatal que nunca llegó y se comenzó un proceso de acaparamiento criminal del territorio y de las economías ilegales que poseían las FARC; (2) en otros territorios no se tuvo esperanza alguna de que llegaría la oferta estatal, puesto que se dio con gran rapidez la apropiación territorial y de economías ilegales por parte de organizaciones criminales y grupos armados ilegales, como sucedió en el Pacífico nariñense, donde grupos disidentes de las FARC, grupos armados organizados y el ELn se disputaron el control del territorio, y (3) otros municipios con antigua presencia de las FARC no tuvieron una presencia integral del Estado ni arribaron grandes estructuras violentas o de crimen organizado, sin embargo sí se incrementaron los índices de inseguridad a causa de la estructuración de grupos dedicados a la delincuencia común (Fundación Paz y Reconciliación, 2019b, p. 28).

Entre los actores de mayor relevancia en la situación de orden público actual se encuentran, por supuesto, los grupos post-FARC. Estos se derivaron directamente de las antiguas estructuras guerrilleras desmovilizadas que durante las negociaciones, o ya en proceso de posconflicto, decidieron desertar 
y optaron por continuar la lucha armada para la consecución del poder, aunque al parecer están más comprometidos con las rentas ilícitas del narcotráfico que con la lucha revolucionaria (McDermott, 2018). Su número se estima entre 800 y 1.600 personas (Fundación Paz y Reconciliación, 2019a), aunque InSight Crime (2018) habla de 2.500, y operan en las zonas de antigua ocupación de las FarC, especialmente en los municipios donde existe un gran porcentaje de cultivos ilícitos, de modo que tienen mayor incidencia en los departamentos de Nariño, Cauca, Caquetá, Guaviare, Vaupés, Guainía y Meta. Lo anterior socava las condiciones de seguridad territorial y las garantías por parte del Estado, por lo cual constituye un gran reto para la implementación material de los acuerdos, al igual que para la presencia estatal y el control territorial (International Crisis Group, 2017).

Aproximadamente el $80 \%$ de estos grupos está compuesto por excombatientes de las líneas de las Farc. Sin embargo, difieren mucho en términos organizacionales a la antigua guerrilla y entre ellos mismos, como los mandos, las estructuras jerárquicas, las motivaciones que arguyen en los discursos y comunicados, al igual que la cohesión, magnitud y acciones de violencia; cambios que se observan principalmente a nivel subregional (Fundación Paz y Reconciliación, 2019a). No obstante, las grandes facciones de disidentes siguen funcionando como lo han hecho históricamente, combatiendo los esfuerzos estatales de erradicación de coca, controlando los corredores de tráfico, efectuando ataques selectivos contra las fuerzas de seguridad del Estado y, en general, resolviendo disputas y garantizando el orden público a nivel local donde tienen legitimidad (International Crisis Group, 2017).

De otro lado, también existen grupos que se separaron del proceso sin alegar alguna razón ideológica o política, sino que decidieron permanecer en los negocios de las rentas ilegales, principalmente el narcotráfico, y no pretenden ser necesariamente un agente regulador de la vida social en el territorio. Estas características son más comunes en los grupos post-Farc. Estos grupos han sido denominados como FARCRIM o FARC Criminales, según Jeremy McDermott (2018), y han continuado desarrollando sus actividades criminales donde tradicionalmente han operado. Son grupos de diverso tamaño que comprenden desde pequeñas agrupaciones de pocos hombres hasta poderosas 
estructuras de cientos de miembros, las cuales están conformadas no solo por exguerrilleros y milicianos, sino además por pandillas juveniles y delincuentes en zonas urbanas que la extinta organización subcontrataba para labores criminales - grupos que quedaron a la deriva con la salida de las FARC de la escena criminal- Inclusive algunos grupos cuentan con presencia de exparamilitares que cooperan con sus antiguos enemigos con la lógica del lucro ilegal (InSight Crime, 2018).

Estos grupos se han convertido de manera acelerada en uno de los grandes promotores de violencia en los conflictos criminales que tienen lugar en los variados territorios de Colombia. Esto sucede especialmente en Nariño, donde los grupos Guerrillas Unidas del Pacífico (GUp), Frente Oliver Sinisterra y Gente de Orden se han esforzado en ampliar su presencia territorial y el número de efectivos, de manera que han acaparado zonas rurales y urbanas desde el municipio de Tumaco y por todo el litoral pacífico hasta Buenaventura. Se trata de estructuras que están integradas por antiguos milicianos del Frente 29 y Daniel Aldana que operaban en el Pacífico, además del reclutamiento de nuevos integrantes por medio de los incentivos económicos. Inclusive, en ocasiones se han presentado enfrentamientos entre estos tres grupos con el fin de asegurar eslabones de las rentas ilícitas de las drogas en el territorio (Fundación Paz y Reconciliación, 2018).

Para finalizar, entre los grupos post-FARC se encuentran las organizaciones que apoyaron a dicha guerrilla con logística e inteligencia — dirigiendo redes de financiamiento y economías ilegales, así como penetrando entes estatales - y que siguen siendo leales a las causas políticas del movimiento, pero que no están contabilizadas en las cifras oficiales, puesto que sus labores en el interior de la organización eran en mayor medida de dirección y administración financiera, sin que tuvieran mayor impacto material en el terreno. Estos grupos, denominados como FARC ocultas se pueden identificar, por ejemplo, en zonas donde no han existido cambios incrementales de violencia, en áreas de tradición cocaleras de Putumayo y Caquetá, pese a que en estos departamentos se producen aproximadamente cien toneladas de cocaína al año. Esto se debe a que no ha existido cambio alguno en el tráfico de drogas, pues sigue siendo administrado por los mismos actores (InSight Crime, 2018; McDermott, 2018). 
Estos grupos post-FarC sacan réditos de las bases y apoyos construidos durante el conflicto para mantener el control y la expansión territorial, con lo cual garantizan su actividad criminal en las rentas ilegales. Sin embargo, pese a que en el pasado pertenecían a un misma organización, actualmente no constituyen una estructura única con líderes identificables, como ocurría en el pasado con la configuración de secretariado que tenía las FARC-Ep (International Crisis Group, 2017).

Por supuesto, el ELN sigue siendo un actor clave en este complejo escenario y, de hecho, debido a la reacomodación de fuerzas se ha estado consolidando como el actor más importante de la primera etapa del narcotráfico colombiano, especialmente a lo largo de la costa del Pacífico (Chocó, Cauca y Narińo) (McDermott, 2018). El ELn no podrá reemplazar a las FARC en todo el país, ya que no cuenta con el mismo alcance nacional. Sin embargo, tanto en la costa Pacífica como en la frontera con Venezuela, dos de las áreas de narcotráfico más estratégicas, está ganando poder y comenzando a ejercer hegemonía. Si bien ha habido violencia en la costa Pacífica, donde tradicionalmente las FARC y el ELN han cohabitado con otras organizaciones de narcotráfico, no ha habido mucha violencia en la frontera con Venezuela, donde el ELN es el actor más fuerte y donde encuentra pocos obstáculos para el flujo de cocaína en la turbulenta nación vecina (McDermott, 2018).

Esta descripción tiene el propósito de poner en evidencia que la consolidación de una paz territorial está muy lejos de ser una realidad en el país. Los actores armados han identificado correctamente que el Estado colombiano no tiene las capacidades para llegar efectivamente a los territorios (al menos no en lo inmediato) con proyectos y programas de desarrollo social, pero tampoco se ha decidido por una línea clara frente a la implementación o no del acuerdo de paz, condicionado, como puede ser evidente, por la posición política del actual gobierno. Esta esquizofrenia entre implementar y no implementar lo pactado ha abierto ventanas de oportunidad para que estos actores armados se posicionen y se afiancen en espacios que alcanzaron a ver la posibilidad de consolidar espacios de paz, pero la perdieron. 


\section{La reforma al sector seguridad y la paz en los territorios}

La reforma del sector seguridad (Rss) se debe entender como toda la plétora de acciones conducentes a la creación de esquemas de buen gobierno de la seguridad; la efectividad y eficiencia en el suministro de la seguridad y la justicia; la transformación y redefinición de las funciones de las fuerzas de seguridad; la construcción y el mejoramiento de las relaciones cívico-militares/ policiales; el mejoramiento de las capacidades de civiles de influir en debates de seguridad y defensa, todo dentro de un marco democrático.

Sin embargo, es importante señalar que los procesos de reforma al sector seguridad no ocurren en un vacío contextual, sino que en múltiples ocasiones (como se evidencia en otros capítulos del libro) son el resultado de transiciones y procesos de estabilización. Para el caso colombiano, los procesos de Rss están, por supuesto, anclados a la etapa de posacuerdo que actualmente vive el país. Para diversos analistas, el posconflicto y la construcción de la paz, además de involucrar acciones y prácticas como la justicia transicional, el desminado, los procesos de Desarme, Desmovilización y Reincorporación (DDR), el rediseño de instituciones y los procesos de reconciliación y reparación a las víctimas, también implican la transformación de los sectores de seguridad y defensa, en el entendido de que la seguridad deja de ser comprendida, de manera exclusiva, como la seguridad del Estado (seguridad nacional) y transita hacia una visión de la seguridad como un derecho que tienen los individuos de gozar de libertad frente a la necesidad y frente al miedo (seguridad humana) (Schnabel \& Ehrhart 2005; Schroeder et al., 2014; Sedra, 2006, 2017).

En una etapa de posconflicto, las fuerzas de seguridad del Estado están llamadas a revisarse y reorientar sus acciones con miras a las nuevas realidades nacionales. De manera concreta, la Policía Nacional ha estado realizando una serie de proyectos con el propósito, precisamente, de reformarse para respetar su mandato constitucional (brindar garantías que permitan el ejercicio de la convivencia armónica de las poblaciones) con respecto a otro tipo de realidades, más cotidianas, que afectan el diario vivir de los ciudadanos del país. En términos bastante amplios, los ajustes que está haciendo la Policía en el 
periodo de posacuerdo tienen como objetivo recuperar el carácter civil de la institución, que durante varias décadas se había desviado hacia tendencias de corte militar como consecuencia de las dinámicas propias del conflicto armado. Este carácter militarizado de la Policía le ha impedido, hasta el momento, tener un abordaje integral de los problemas de las comunidades desde una óptica de convivencia ciudadana.

Sin embargo, a pesar de estas iniciativas — que han surgido en el seno de las mismas instituciones y no mediante una coordinación clara y dirigida de la estatalidad_- existe una problemática que es precisamente el núcleo del presente capítulo, es decir, la territorialidad. Diferentes analistas han puesto de presente que pese a la descentralización administrativa que existe, en Colombia se gobierna desde el centro y para las grandes ciudades, de manera que se deja de lado la mayoría del territorio nacional. Como se mencionó anteriormente, gran parte de la geografía de Colombia se encuentra en un estado de apartheid institucional, con lo cual se hace referencia a que las poblaciones que habitan estas zonas son discriminadas, pues sus derechos no son reconocidos debido a la debilidad estatal. Como lo sugieren explícitamente García y Espinosa (2016, p. 7), "si en Suráfrica existía una segregación fundada en la prevalencia de una raza, en Colombia existe una segregación fundada en la prevalencia de unos territorios sobre otros".

Por supuesto, esta situación tiene fuertes repercusiones también en el ámbito de la seguridad. Esto pareciera ser contradictorio, pues se ha vuelto un lugar común sugerir que aunque el Estado colombiano no llega con toda su institucionalidad, al menos sí lo hace con su Fuerza Pública. Sin embargo, la situación no es alentadora cuando se revisa en qué medida las necesidades en materia de seguridad y protección de las comunidades coinciden con la oferta que brindan las instituciones de seguridad y defensa. Por el contrario, lo que se aprecia es que estas instituciones han puesto un énfasis especial en la lucha a los grupos al margen de la ley y en programas, proyectos y directrices preestablecidas desde el centro nacional, aplicables a toda la geografía, con lo cual han descuidado la atención a las problemáticas de seguridad y convivencia que afronta la ciudadanía y para las cuales las instituciones no tienen capacidad operativa ni cobertura. Esta situación se empeora por el hecho de que estas 
políticas de talla única enfocadas en los datos duros tienden a ser inmediatistas y a valorar positivamente los impactos que se pueden mostrar a la opinión pública, de manera que las políticas de prevención de las violencias y promoción de la convivencia pacífica no hallan asidero sustancialmente porque no son medibles y, por consiguiente, no son mostrables ni vendibles.

\section{Retos de la Fuerza Pública de cara al posacuerdo}

En un escenario de construcción de paz se hace indispensable que la seguridad como derecho de los ciudadanos sufra un cambio radical. En ese sentido, es determinante que las instituciones de seguridad y defensa del país generen estrategias de seguridad con enfoque territorial. Con base en el diagnóstico de los argumentos que presenta la Fundación Ideas para la Paz (2016a, 2016b) en diversos informes y el análisis de la revisión documental sobre una estrategia del Modelo de Construcción de Paz de la Policía Nacional (2019), orientada hacia la consolidación de espacios de convivencia en Colombia, es posible argumentar que los cambios que se deben generar tocan asuntos políticos, técnicos, presupuestales, procedimentales y conceptuales. A modo de síntesis, estos se podrían agrupar en siete problemas/retos que es necesario abordar:

1. Falta de voluntad política de las autoridades político-administrativas de los territorios para abordar de manera integral los asuntos de seguridad y convivencia de las comunidades.

2. Presupuestos muy limitados para tratar asuntos de seguridad y convivencia en los territorios.

3. Mal funcionamiento de los espacios dedicados a las discusiones de convivencia y seguridad ciudadana y mala utilización de los fondos.

4. Falta de coordinación interinstitucional y de articulación institucional.

5. Existencia de indicadores de impacto que desincentivan el desarrollo de estrategias integrales de convivencia y seguridad ciudadana.

6. Ausencia de metodologías claras (tanto de Policía como de Fuerzas Militares) que permitan abordar estas temáticas. 
7. Poca preparación del personal para hacer frente a las demandas en materia de seguridad y convivencia ciudadana.

En primera instancia, si bien las autoridades locales y de manera concreta el alcalde, como primera autoridad, son los responsables en materia de seguridad y orden público, lo que se evidencia en los territorios es que estas autoridades no tienen incentivos reales para abordar de forma integral los asuntos de seguridad ciudadana y convivencia. Esto ocurre sustancialmente porque a medida que se hacen evaluaciones de sus mandatos, tanto a nivel local como nacional, lo importante resulta tener evidencia medible de que ciertos temas de seguridad han mejorado, por ejemplo que los homicidios y los robos han disminuido. Debido a esta preocupación por los indicadores se tiende a dejar de lado todo un ámbito de problemáticas diarias en las comunidades y de comportamientos que afectan la convivencia, cuyo abordaje no es reactivo por medio de la fuerza ni la intervención directa de las capacidades de policía, sino por medio de estrategias integrales que prevengan la violencia y promuevan la convivencia.

Como lo sugieren Bulla y Guarín (2016a), el gasto en materia de seguridad depende de la capacidad fiscal de los municipios, lo que genera importantes diferencias entre lo urbano y lo rural, donde estos últimos tienen menos capacidades (en términos de personal, equipamiento e instalaciones) para abordar las problemáticas de seguridad. Las fuentes de financiación son dos fondos, principalmente, los Fondos Territoriales de Seguridad y Convivencia Ciudadana (Fonset) y el Fondo Nacional de Convivencia y Seguridad Ciudadana (Fonsecon). A juicio de los autores y a partir del diagnóstico de la Policía, estos recursos no son suficientes porque para el caso del FONSET es necesario realizar contratos de obras públicas, situación que no es muy frecuente en los municipios más rurales y apartados, con lo cual este fondo sería virtualmente inexistente. En el caso del Fonsecon, el gasto de estos recursos depende de las prioridades establecidas por las autoridades, que, como se sugirió en el punto anterior, no siempre coinciden con las necesidades en materia de seguridad y convivencia de las poblaciones. Esta situación es bastante paradójica, teniendo en cuenta que en los territorios más rurales y, por lo tanto, más extensos con 
poblaciones dispersas, la seguridad tiende a ser un rubro que genera más costos y por ende requiere de mayor personal, de mejor equipamiento en términos de movilidad y comunicaciones, pero ocurre todo lo contrario.

El tercer punto, que se desarrolló en parte más arriba, tiene que ver con la utilización de recursos que, en algunos casos, son inexistentes y en otros son dedicados a otras actividades. A esto habría que sumarle, por supuesto, temas de corrupción. Con respecto a los espacios destinados a la discusión de los temas de seguridad y convivencia ocurre que si bien estos se encuentran regulados por ley, como son los Consejos de Seguridad y los Comités Territoriales de Orden Público, funcionan en un escenario de escasez presupuestal, descoordinación y agendas diferenciadas. Una de las principales herramientas con las que disponen las autoridades político-administrativas son los llamados Planes Integrales de Seguridad y Convivencia Ciudadana (PISCC), los cuales son documentos de política pública mediante los cuales los alcaldes y demás involucrados en temas de convivencia y seguridad planifican cuáles serán las estrategias que se desarrollarán para abordar estos retos. Sin embargo, como lo sugieren Bulla y Guarín (2016a), y como fue destacado a partir de la experiencia de la Policía, estos planes terminan siendo sobre todo un requisito legal para los alcaldes y no una herramienta de planeación estratégica que permita priorizar problemáticas y optimizar recursos. En algunos casos se ha visto que estos planes son elaborados por consultores externos, sin prestar atención a las reales problemáticas territoriales. En otros casos más problemáticos se ha visto cómo ciertos municipios copian los Piscc de otros territorios.

Un cuarto tema crucial que ha impedido el abordaje de las problemáticas de seguridad ciudadana y convivencia con enfoque territorial tiene que ver con la falta de gestión territorial, coordinación interinstitucional y articulación institucional. Al respecto, se observa que estos mecanismos obedecen a una lógica según la cual abordar de manera preventiva la convivencia y la seguridad ciudadana requiere del apoyo conjunto de otras instituciones y se hace indispensable gestionar recursos y alianzas para que esto se pueda lograr. La implementación de procesos de prevención con las comunidades y la promoción de la convivencia hacen necesario que las autoridades políticoadministrativas (gobernadores y alcaldes) asuman su función de Policía. Esto 
implica intervenir de manera directa las causas y los factores estructurales originadores de delitos, comportamientos contrarios a la convivencia y violencias, tales como la falta de alumbrado público, la infraestructura vial, la educación, la salud y el bienestar de las comunidades, entre muchos otros. La falta de intervención oportuna de estos riesgos sociales ha ocasionado que tanto el Estado como la Policía Nacional se vean involucrados en acciones de reacción y control frente a los fenómenos que afectan la seguridad ciudadana.

Sin embargo, estos conceptos, provenientes de la administración pública e interiorizados desde hace décadas dentro de la doctrina policial, han terminado en letra muerta en la mayoría de los casos o como meros formalismos sin aprovechar realmente los beneficios que ofrecen. Muchas veces este desaprovechamiento se debe, desde el lado policial, a las exigencias de cumplimiento y tareas que vienen del nivel central (direcciones), el cual emite órdenes directas que dan poco espacio a la articulación local con las estaciones y sus necesidades reales en materia de convivencia y seguridad ciudadana.

Un quinto punto de vital importancia y que tal vez impacta directamente en otras de las falencias tratadas más arriba tiene que ver con el tipo de indicadores que son utilizados actualmente para medir las estrategias de Policía. Una de las consecuencias que indirectamente ha generado el conflicto armado en Colombia tiene que ver con la necesidad de generar constantemente reportes inmediatos y mediáticos sobre las condiciones de seguridad del país. Como consecuencia se ha generado un "aperezamiento" por parte de la institucionalidad responsable (Ministerio de Defensa, Ministerio del Interior, Fuerzas Militares y Policía) en generar indicadores que permitan visibilizar otro tipo de dinámicas más allá de los hechos delincuenciales y de violencia más obvios como los homicidios, las masacres, los hurtos, las riñas, entre otros.

Cuando un conflicto armado concluye, o por lo menos genera un cerramiento temporal, se abren otras formas de inseguridades y retos que deben ser abordados desde ópticas sustancialmente diferentes. Entre estos retos se encuentra la gestión de la conflictividad social natural presente en las comunidades y otras dinámicas que se desprenden de los procesos de desarme y reintegración a la vida civil de los excombatientes. En últimas, se trata de la re-visibilización de otro tipo de conflictividades frente a las cuales 
no solo son necesarias nuevas herramientas y metodologías, sino también nuevas formas de medirlas. Si bien la Policía tiene establecido dentro de su doctrina un procedimiento denominado Prevención y Convivencia, este carece de contenido. No solo no existen metodologías apropiadas para cumplir estos objetivos, sino que tampoco existen indicadores adecuados para hacer seguimiento. Por este motivo, cuando las estaciones en las zonas más rurales deben hacer tareas de prevención se enfrentan a una encrucijada real entre hacer prevención y hacer vigilancia, pues esta última es la única que puede ser medida con los indicadores de impacto existentes.

Estrechamente relacionado con el punto anterior, un importante reto que tienen las instituciones de seguridad y defensa de cara a un proceso de estabilización territorial es crear metodologías que permitan el abordaje diferencial de las problemáticas que emergen en lo local. Si bien mayoritariamente esto impactaría únicamente a la fuerza policial, es importante destacar que, dada la presencia de actores armados en diversos territorios de Colombia, como se mostró anteriormente, los militares siguen conviviendo en espacios donde también habitan comunidades.

Como lo ponen en evidencia Bulla y Guarín (2016b, p. 30), con las Fuerzas Armadas que siguen ejerciendo control territorial en ciertas partes del país se presentan dos situaciones que afectan la provisión de medidas de seguridad y convivencia. Por un lado, sugieren que, debido a la supremacía militar (dadas las condiciones de orden público), las autoridades locales y sus instituciones tienen dificultades para ingresar en ciertas comunidades para brindar los servicios institucionales propios de la esfera civil, así como para hacer diagnósticos efectivos en términos de las necesidades en materia de seguridad y convivencia ciudadana. Por otro lado, esta misma presencia de las Fuerzas Militares se ha vuelto un originador de problemas de convivencia en los territorios, ya sea porque los habitantes perciben su presencia como una potencial fuente de amenaza por parte de actores armados, bien porque se han hecho públicas ciertas dinámicas de convivencia entre miembros de la Fuerza Pública y bandas criminales, lo cual, claramente, afecta la tranquilidad de las comunidades. Es evidente que retirar a las Fuerzas Militares de ciertas zonas sensibles de la geografía no es una opción y que, por el contrario, es 
un requerimiento que se fortalezca la presencia militar frente al crecimiento y aumento de los hostigamientos por parte de los actores armados ilegales. Sin embargo, sí es deseable que el Ministerio de Defensa y el estado mayor de las Fuerzas Militares establezcan cierto tipo de mecanismos que permitan generar un interrelacionamiento diferenciado con las poblaciones de estos territorios, el cual se debe caracterizar por comportamientos que van más allá de la mera autoridad militar sobre la población civil y que, por el contrario, favorezcan la confianza de un lado hacia el otro.

Algo similar ocurre del lado policial. Situación que, sin embargo, tiende a ser más acuciante si se tiene en cuenta que el rol constitucional y misional de la Policía consiste, precisamente, en garantizar la convivencia pacífica de los ciudadanos del país. Como se comentó más arriba, si bien existen procesos y procedimientos orientados hacia el cumplimiento de este objetivo, en la práctica solo existe una guía ${ }^{3}$ bastante escueta y esquemática, que si bien les dice a los policías "qué hacer", no les dice el "cómo". Igualmente, el nivel de preparación y formación policial en función de construir convivencia también es bajo, teniendo en cuenta que la convivencia se construye a partir de la generación de confianza entre las partes, para lo cual es necesario desarrollar habilidades blandas, tales como la comunicación, el liderazgo y la gerencia de la convivencia ciudadana (Policía Nacional, 2016, p. 34).

Este, precisamente, es el último reto que deben enfrentar las instituciones de seguridad y defensa, y con mayor énfasis la Policía de cara a un escenario de posacuerdo. Tal vez este constituya uno de los puntos más problemáticos, pero a la vez más importantes en lo que se refiere a la reforma policial, no solo en Colombia sino en otros países de la región. Dado el carácter del conflicto armado en el país, la Policía asumió un rol marcadamente militar, que además de afectar las acciones que desarrollaba durante el conflicto, también impactó duramente su estructura organizacional y el régimen de formación y carrera. Por supuesto, esa formación de corte militarista que ha asumido la institución ha afectado contundentemente el relacionamiento con las comunidades, que perciben a la institución como antagónica a sus intereses, y al final ha

3 Metodología de articulación en prevención para la convivencia y seguridad ciudadana (Policía Nacional de Colombia, 2018). 
deteriorado la imagen y legitimidad de la institución (Bulla \& Guarín 2016b, pp. 51-50). Con esto no se quiere insinuar que la Policía no ha llevado a cabo importantes procesos de profesionalización y formación a lo largo de su vida institucional, especialmente con las reformas de 1993 y 2003, cuando se hicieron modificaciones a la estructura del sistema de formación policial general. El punto va a que de cara a abordar temas de seguridad y convivencia ciudadana, se hace indispensable "ayudar a pasar de una formación policial castrense y en modalidad internado, a una formación policial cívica y con base en el contacto con la comunidad” (Bulla \& Guarín, 2016a, p. 59).

Estos puntos constituyen, a grandes rasgos, las principales problemáticas que deben encarar las instituciones de seguridad y defensa (con un énfasis especial en la Policía Nacional) para afrontar y gestionar las dinámicas del posacuerdo en los territorios. Algunos de estos elementos han sido abordados recientemente a través de proyectos orientados a la reforma policial, con la colaboración y financiación de la cooperación internacional. En lo que resta del capítulo se describe de forma general una de estas experiencias de la Policía, la cual se gestó con apoyo del fondo multidonante de Naciones Unidas y fue ejecutada por la Organización Internacional para las Migraciones (Oıм) con sede en Bogotá entre 2018 y 2019. Por último, se exponen algunas de las reformas de carácter más estructural que serían necesarias para abordar de forma efectiva la seguridad y la convivencia en clave territorial.

\section{SUMA: voces y acciones para la convivencia}

Dentro del contexto de posacuerdo, la Policía Nacional desarrolló una poderosa herramienta para prepararse frente a los desafíos que implicaba un periodo de transición, donde las acciones, los procesos y los procedimientos que tenía establecidos no le permitían cumplir los objetivos que como institución se planteaba. Esta herramienta consistió en un Modelo de Construcción de Paz, el cual está articulado con el plan estratégico institucional y con el Plan de Modernización y Transformación Institucional (MTI). Dicho modelo se elaboró de manera participativa, incluyendo una encuesta diligenciada por más de 125.000 policías y la participación directa de más de 400 policías en 
espacios de diálogo constructivo. La metodología utilizada estuvo orientada a comprender, desde el conocimiento y la experticia interna de la institución, cuáles eran los ejes que debía fortalecer la Policía Nacional para hacer frente a los retos del momento histórico de Colombia. Con base en la información recolectada y luego de un proceso metodológico riguroso, se definieron los enfoques y los componentes que constituyen el modelo. Para materializarlo se implementó una lógica de programa, en la cual se establecieron 18 proyectos que buscaron desarrollar operativamente cada uno de los componentes con una orientación de fortalecimiento institucional, es decir, que su implementación debe generar productos que deriven en la ampliación de capacidades de la institución.

En relación con los componentes de "Gestión territorial de la seguridad y convivencia” y "Cultura y pedagogía de paz", se elaboró un proyecto orientado a transformar la relación entre la Policía Nacional, la comunidad y las instituciones locales a través de la planeación participativa de la convivencia. El proyecto recibió el nombre de "Suma: voces y acciones para la convivencia” y se implementó en cinco municipios piloto: Neiva (Huila), corregimiento de San Luis; Icononzo (Tolima), vereda Paticuinde; La Montañita (Caquetá), inspección de Santuario; Mitú (Vaupés), comunidad indígena del Doce de Octubre, y Aracataca (Magdalena), corregimiento de Sampués (Policía Nacional, 2019).

El proyecto implicó una serie de fases de trabajo con las estaciones de Policía, las comunidades y las autoridades político-administrativas de los municipios y territorios priorizados. Sin embargo, en términos generales la intervención pretendió desarrollar un cambio en la forma de hacer policiamiento en los territorios, con énfasis en la ruralidad. En ese sentido, las acciones que se generaron tuvieron el propósito de establecer una modalidad de policiamiento comunitario (community policing) (Frühling, 2003) que le permitiera a la institución acercarse a la comunidad para entender sus necesidades reales en materia de seguridad y convivencia ciudadana, de modo que fuera capaz de gestionarlas con la participación de las autoridades político-administrativas y otras instituciones y actores de interés. 


\section{Componentes de Suma}

\section{Participación}

Suma operó con la lógica de consolidarse como un cambio permanente dentro de la Policía, fortaleciendo los procedimientos de convivencia y prevención que, como se ha descrito en secciones anteriores, no han sido desarrollados de manera apropiada por ese sesgo puramente policivo de la institución. Pero además de ello, y con el propósito de consolidar un servicio de policía realmente responsivo para con las comunidades, el componen participativo de Suma fue fundamental.

La institución policial ha involucrado tradicionalmente a la ciudadanía en la gestión de la seguridad como una fuente de información frente a la comisión y presencia del crimen y el delito. Si bien esto corresponde a un comportamiento usual en las policías del mundo, no obedece plenamente a la manera como la comunidad debe ser incluida en sociedades democráticas y en procesos de transición para abordar, de modo efectivo, temas de seguridad y de convivencia. Un servicio de policía orientado hacia la prevención no puede "hacer uso" de la comunidad de manera utilitaria, sino que se requiere una participación real de la sociedad para generar un tejido social mucho más denso y sólido que permita, en primer lugar, evitar y prevenir las conductas contrarias a la convivencia. De hecho, estos mecanismos y espacios de seguridad participativa y comunitaria parten de la premisa de que la población local dispone de una serie de información y conocimiento clave para los policías, pero más allá de esto se fundamentan en un nuevo tipo de relacionamiento entre la policía y la comunidad que se vuelve clave para el éxito de este tipo de intervenciones.

En el marco del proceso de reforma de la Policía colombiana, la inclusión de la ciudadanía ha transitado por diferentes estadios, en los cuales la participación ciudadana ha tenido cada vez mayor relevancia. Desde los Modelos de Participación Comunitaria (PARCo) hasta el más reciente Modelo Nacional de Vigilancia Comunitaria por Cuadrantes (MNVCC), el enfoque de participación ha sido evidente e indiscutible. Sin embargo, estos modelos ven al ciudadano y las comunidades como facilitadores de información de conductas y hechos que les sirven a los policías para ejecutar las funciones propias del servicio. Es decir, la participación ciudadana ha tendido a ser artificial, en la medida en que 
se concibe a los ciudadanos como informantes, útiles para ciertos momentos, pero que no se integran en el servicio de Policía como tal. Por supuesto, esta forma de inclusión es supremamente útil ya que, como se vio, la comunidad dispone de una serie de información relevante para la planeación del servicio de Policía. Pero no es suficiente para generar una genuina participación.

La importancia de lograr una efectiva participación comunitaria en la gestión de la seguridad y la convivencia radica en tres puntos fundamentales (González, 2016). Por una parte, el efectivo involucramiento comunitario es una ruta eficiente para que "lo local" se comunique con "lo nacional". En gran medida las políticas públicas de seguridad tienden a ser implementadas del centro hacia la periferia, sin prestarles atención a las verdaderas necesidades territoriales. En ese sentido, con el involucramiento comunitario se generan espacios de intercambio de información sobre las demandas y las necesidades locales que van a impactar en la manera como las instituciones, las políticas, las estrategias y los recursos se disponen y despliegan en los territorios.

En segunda medida, la efectiva participación comunitaria sirve como un espacio informal para que la Policía rinda cuentas a la comunidad. Si la Policía es representativa y sirve a los intereses de la comunidad, esta la percibirá de forma positiva y cimentará otro tipo de relacionamiento con la institución. Si bien esta es la lógica normal del funcionamiento de las políticas públicas, en lo que se refiere a la seguridad — y en un escenario como el colombiano- las dinámicas de seguridad y convivencia tienen comportamientos heterogéneos dependiendo del territorio, de sus dinámicas sociales, de la manera como se insertó el conflicto armado y de cómo ha evolucionado, entre otros factores. Por ello, Suma fomentó la escucha local frente a los problemas de seguridad y convivencia, un aspecto clave que legitima no solo su accionar, sino también el de las demás instituciones participantes.

Por último, la efectiva participación comunitaria en el abordaje de temas de seguridad y convivencia va a tener un efecto muy positivo en reducir la práctica, muy usual en América Latina, de generar justicia por sus propios medios (González, 2016). No solo esto, sino que además aquellos ciudadanos que perciben que sus comportamientos contrarios al bienestar comunitario no están siendo controlados ni castigados, no tendrán ningún tipo de incentivo 
para evitar cometerlos. Cuando la comunidad se ve involucrada de manera efectiva, no podrá dejar pasar ese tipo de conductas y serán sujeto de control social y, posteriormente, institucional-policial.

En este marco, Suma operó mediante la promoción de espacios participativos en los cuales las comunidades fueron involucradas de modo directo en la gestión de su propia seguridad y convivencia. Con esto, no solo se favoreció la planeación del servicio de Policía al contar con información clave surgida del seno de la comunidad y sus necesidades, sino que al mismo tiempo la comunidad se benefició de poder contar con la institucionalidad policial para abordar sus problemas reales. De igual modo, en el trasfondo de estos encuentros se desarrolló un vínculo "más real" entre la comunidad y la Policía, un vínculo que no se caracterizó por el utilitarismo, sino por el real y genuino contacto entre individuos.

\section{Convivencia y prevención}

La prevención comprende un enorme universo de enfoques que se han consolidado a partir del desarrollo teórico-práctico en las áreas de la salud, el desarrollo social y la seguridad. Sus propuestas en prevención se han constituido como marcos de referencia con alcances prácticos, lo cual les ha permitido a las diferentes instituciones que adoptan enfoques preventivos trazar rutas de acción con programas y estrategias para proteger la vida, los bienes y el bienestar de las poblaciones.

La prevención relacionada específicamente con el tema de la seguridad - es decir, la prevención del delito y las violencias - se enmarca en el proceso de consolidación de una forma de seguridad entendida como la garantía de protección, convivencia y tolerancia interpersonal en el marco de una comunidad política, esto es, de la seguridad ciudadana. Las políticas preventivas orientadas a la protección de la seguridad ciudadana son tareas estatales complejas, en cuanto requieren de la articulación de múltiples y diferentes actores sociales, formales e informales, y en las cuales tiene enorme influencia el liderazgo político del poder ejecutivo.

La apuesta, en el marco de la reforma policial de Suma, le apuntó a superar la idea de prevenir exclusivamente el crimen y el delito, para direccionarse hacia 
la prevención de comportamientos contrarios a la convivencia y a la promoción de actitudes que la favorezcan. Cuando la Policía orienta su trabajo hacia el fomento de la convivencia, lo preventivo se torna determinante. Precisamente, si se tiene en cuenta que los conflictos suelen surgir de pequeñas diferencias que luego escalan a formas de violencia insospechadas, se comprende que se trata de una razón más que válida para reorientar los esfuerzos institucionales hacia este aspecto de la condición humana: el conflicto cotidiano. Dicho de otra forma, la gestión de la convivencia cotidiana.

Es así como Suma materializó este objetivo, abordando la convivencia pacífica, sus causas y sus factores incidentes. Se partió de un encuentro de voces, el cual permitió el diálogo sobre las posibilidades de unos y otros actores, empoderando a la comunidad para que resolviera muchos de los asuntos problemáticos (prevención comunitaria), surgieran espacios de co-construcción de iniciativas que pudieran transformar situaciones propicias para la violencia y el crimen (prevención situacional) y se generaran compromisos entre las instituciones y las organizaciones encargadas del control social y la solución de problemas sociales (prevención social).

\section{Relaciones Policía-comunidad}

Suma operó con la lógica del policiamiento comunitario. Es decir, como una filosofía que promueve estrategias organizacionales que apoyan la generación sistemática de partnerships (entre diversos actores) y utilizan técnicas de resolución de problemas, de modo tal que se puedan abordar de forma eficiente y efectiva las necesidades comunitarias tales como el crimen, el desorden social y el miedo (Reisig \& Kane, 2014). En ese sentido, un objetivo central de la experiencia consistió en mejorar las relaciones entre la Policía y la comunidad. Sin embargo, lejos de ser una estrategia de relaciones públicas para mejorar su imagen - como algunos autores han sugerido (Wisler \& Ihekwoaba, 2009)_, la propuesta de Suma reivindica el policiamiento comunitario, en la medida en que se trata de una estrategia surgida en el marco de un modelo de construcción de paz que le apunta, de manera genuina, a la reconstrucción de lazos y tejido social entre los miembros de las comunidades, y entre estas y las demás instituciones del Estado encargadas de proveer seguridad y convivencia territorial. 
Por medio del enfoque comunitario de SumA, esta mejora en el relacionamiento entre las comunidades y la Policía se reflejó en ambos actores de forma independiente y en el trabajo conjunto. En relación con la comunidad, los resultados permitieron evidenciar que la metodología de trabajo implementada fomenta el desarrollo de mayor legitimidad de la Policía hacia la comunidad, dado el reconocimiento del alcance de su servicio y la mayor satisfacción con este por parte de la comunidad. Aunque aún no se puede concluir si este aumento en la legitimidad es producto del contenido de las actividades, de la proximidad del policía o de ambas, lo que sí muestran los resultados es que luego de la implementación de la metodología existe una mayor disposición de la comunidad a colaborar con la Policía en su labor.

Asimismo, la implementación de la metodología ha permitido establecer que las personas que recibieron las actividades y trabajaron de manera conjunta con la Policía en la gestión de la convivencia tienen una mejor percepción de seguridad que las que no lo hicieron. Este dato es significativo, si se tiene en cuenta que los delitos y los comportamientos contrarios a la convivencia son una situación objetiva del territorio. Así pues, ante los mismos hechos, las personas que trabajaron de manera cercana a la Policía se sienten más seguros que los que no lo hicieron.

Por su parte, los policías que implementaron la metodología Suma también transformaron su manera de relacionarse con la comunidad, a partir tanto de las capacitaciones que se realizaron en el proceso, como del efecto transformador propio del diálogo abierto y genuino con la comunidad. En este sentido, algunos de los principales aspectos en los que se evidencia este proceso de cambio es que varios policías que implementaron la metodología manifestaron el valor de comprender a la comunidad como coautora y/o corresponsable de su propia gestión de la seguridad, la prevención y la convivencia. Esta visión, además de estar acorde con el mandato constitucional y misional de la Policía Nacional, también se conecta con la horizontalidad que propone el concepto mismo de policiamiento de "civiles que cuidan civiles" y de policía comunitaria.

Finalmente, tal vez el mayor impacto en la transformación de la relación entre la Policía y la comunidad está en la capacidad y las habilidades para 
trabajar de forma articulada. Suma permitió evidenciar una mayor disposición para la participación, que se materializó en la identificación de puntos en común entre la Policía y la comunidad acerca de la convivencia y su gestión, así como en la construcción de una agenda común que distribuyera responsabilidades y posibilitara la consolidación de capacidades para la prevención. A partir del fortalecimiento de esta relación, se abonó el camino para que otras instituciones y organizaciones locales, principalmente la administración municipal, se integrara y aportara a la solución participativa de situaciones que si bien derivan en problemas de convivencia, tienen causas que no son del resorte exclusivo de la Policía.

Si la Policía puede ver su contrapunto en la ciudadanía y la comunidad en la institución policial, se podrá ir gestando, de manera paulatina, un verdadero tejido social en lo local, contexto a contexto, barrio a barrio, donde el delito no solo va a ser tratado de manera efectiva y eficiente, sino que además se abordarán los factores de riesgo que pueden estar desencadenando esos delitos y los comportamientos contrarios a la convivencia. La mayor cercanía entre policías y comunidades sirve para transformar esos entornos de riesgo en entornos protectores, donde no solamente se prevengan comportamientos contrarios a la convivencia y la seguridad, sino que se fomenten y promuevan aquellos favorables y constructivos.

\section{Conflicto de intereses}

Los autores no reportan ningún conflicto potencial de interés en la elaboración de este capítulo.

\section{Financiación}

Este trabajo fue apoyado por el proyecto de investigación INV-EES 2975 titulado "El panorama del post conflicto en Colombia: Un acercamiento desde la gobernanza y la reforma del sector seguridad", financiado en su totalidad por la Universidad Militar Nueva Granada. 


\section{Referencias}

Bulla, P., \& Guarín, S. (2016a). La seguridad rural en Colombia: Una oportunidad para la consolidación de Estado. En Controlando el territorio y construyendo seguridad y justicia en el posconflicto colombiano (pp. 36-21). Fundación Ideas para la Paz.

Bulla, P., \& Guarín, S. (2016b). Formación policial y seguridad ciudadana. ¿Cómo mejorar el servicio de Policía? En Retos y desafíos de la Policía en contextos de transición hacia la paz (pp. 72-43). Fescol.

Calvo, J. (2005, junio). El enfoque territorial en las politicas públicas [Ponencia]. V Congreso Nacional de Administración Pública, Guatemala. https://www.academia.edu/3498238/ El_Enfoque_Territorial_en_las_Políticas_Públicas

Carvajal, D. M. (2017). Del enfoque territorial, sus características y posibles inconvenientes en su aplicación en el marco del acuerdo de paz. Universitas Estudiantes, 76-57,16. https://cienciasjuridicas.javeriana.edu.co/documents/4/9203095/3722972.+Daniel+Car vajal.pdf/ad83364d-ffe4-2e92-96f17-8b43394e996

Frühling, H. (2003). Policía comunitaria y reforma policial en América Latina. ¿Cuál es el impacto? Centro de Estudios en Seguridad Ciudadana.

Fundación Paz y Reconciliación. (2018). Cómo va la paz. Pacífico. Fpr. https://pares.com.co/ wp-content/uploads/06/2018/Informe-Pacífico.pdf

Fundación Paz y Reconciliación. (2019a). Cómo va la paz. La reestructuración unilateral del acuerdo de paz. FPR https://pares.com.co/wp-content/uploads/11/2018/INFORMECOMO-VA-LA-PAZ1-.pdf\#

Fundación Paz y Reconciliación. (2019b). Más sombras que luces. La seguridad en Colombia a un año del gobierno de Iván Duque. Frr. https://pares.com.co/28/08/2019/ mas-sombras-que-luces-un-analisis-de-seguridad-en-colombia/

García, M., \& Espinosa, J. (2013). El derecho al Estado. Los efectos legales del apartheid institucional en Colombia. Dejusticia. https:/www.dejusticia.org/wp-content/ uploads/02/2014/El-derecho-al-Estado-Los-efectos-legales-del-apartheid-institucionalen-Colombia.pdf

García, M., \& Espinosa, J. (2016). La geografía de la justicia: evaluando la justicia local en el post-conflicto en Colombia. En Controlando el territorio y construyendo seguridad y justicia en el posconflicto colombiano (pp. 20-6). Fundación Ideas para la Paz.

González, Y. (2016). Varieties of participatory security: Assessing community participation in policing in Latin America. Public Administration and Development, 36, 132-143.

Guarín, S. (2016). Dilemas de la paz territorial y de la participación ciudadana. Fundación Ideas para la Paz. http://www.ideaspaz.org/publications/posts/1275

Hironaka, A. (2005). Neverending wars. The international community, weak States, and the perpetuation of civil war. Harvard University Press.

InSight Crime. (2018, marzo 9). La mafia de las ex-FARC: El nuevo actor en el crimen organizado colombiano. https://es.insightcrime.org/colombia-crimen-organizado/la-mafia-delas-ex-farc-el-nuevo-actor-en-el-crimen-organizado-colombiano/ 
International Crisis Group. (2017). Colombia's Armed Groups battle for the spoils of peace. https://www.crisisgroup.org/latin-america-caribbean/andes/colombia/63-colombiasarmed-groups-battle-spoils-peace

Lemaitre, J., \& Restrepo, E. (2019). Law and violence in the Colombian Post-Conflict: StateMaking in the wake of the peace agreement. Revista de Estudios Sociales, 67, 2-16. https://doi.org/https://doi.org/10.7440/res67.2019.01

Llorente, M., Bulla, P., \& Gómez, C. (2016). De la seguridad para la guerra a la seguridad para la paz. Para un debate de las opciones institucionales. Análisis, 24-1 ,1. http://cdn.ideaspaz. org/media/website/document/57210e6f84515.pdf

McDermott, J. (2018). La nueva generación de narcotraficantes colombianos post-Farc: Los invisibles. InSight Crime. https://es.insightcrime.org/wp-content/uploads/03/2018/ La-nueva-generación-de-narcotraficantes-colombianos-post-FARC-Los-Invisibles.pdf

Policía Nacional de Colombia. (2009). Reglamento del servicio de Policía. Policía Nacional.

Policía Nacional de Colombia. (2016). La convivencia en un escenario de posacuerdo: retos y rutas de acción intersectorial. En Controlando el territorio y construyendo seguridad y justicia en el posconflicto colombiano (pp. 38-19). Fundación Ideas para Paz.

Policía Nacional de Colombia. (2018). Metodología de articulación en prevención para la convivencia y seguridad ciudadana. Policía Nacional.

Policía Nacional de Colombia. (2019). Suma: Voces y acciones para la convivencia. Informe de resultados y aprendizajes. Оім.

Presidencia de la República, Departamento Nacional de Planeación, \& Agencia de Renovación del Territorio. (2019). Unidad didáctica. Enfoque y gestión pública territorial. Presidencia de la República. https://colaboracion.dnp.gov.co/CDT/Desarrollo Territorial/ Portal Territorial/NuevosMandatarios/Enfoque y gestion territorial.pdf

Reisig, M., \& Kane, R. (2014). The Oxford Handbook of Police and Policing. Oxford University Press.

Rojas, P. J. (2017). Doctrina Damasco: Eje articulador de la segunda gran reforma del Ejército Nacional de Colombia. Revista Cientifica General José María Córdova, ,(19)15 119-95. https://doi.org/http://dx.doi.org/19006586.78/10.21830

Schnabel, A., \& Ehrhart, H. (2005). Security sector reform and post-conflict peacebuilding. United Nations University Press.

Schroeder, U., Chappuis, F., \& Kocak, D. (2014). Security Sector Reform and the Emergence of Hybrid Security Governance. International Peacekeeping, 230-214,21.

Sedra, M. (2006). European approaches to security sector reform: Examining trends through the lens of Afghanistan. European Security, 15(3), 323-338.

Sedra, M. (2017). Security sector reform in Conflict-Affected Countries: The evolution of a model. Routledge.

Unidad Policial para la Edificación de la Paz [Uniper]. (2017). Modelo de construcción de paz de la Policía Nacional. Policía Nacional de Colombia. https://www.interpeace.org/ 
latinoamerica/wp-content/uploads/sites/7/2017/05/Modelo-de-Construccion-de-PazPolicia-Nacional-de-Colombia-compressed.pdf

Wisler, D., \& Ihekwoaba, O. (2009). Rethinking Police and society: Community policing in comparison. En Community policing. International patterns and comparative perspectives (pp. 1-18). CRC Press. 